\title{
PRODUCTIVIDAD Y EFICIENCIA EN LAS UNIVERSIDADES PÚBLICAS: EL CASO DE LA UNIVERSIDAD NACIONAL DE LA MATANZA
}

PRODUCTIVITY AND EFFICIENCY IN PUBLIC UNIVERSITIES : THE CASE OF THE NATIONAL UNIVERSITY OF LA MATANZA

\author{
Martínez, D.E. ${ }^{1}$
}

Universidad Nacional de La Matanza

1 Daniel Eduardo Martínez es Doctor en Ciencias Económicas y Magister en Administración de Negocios. Participante en la actualidad del Grupo de Investigación iMARKA, con el código G000705 del catálogo de investigación de la Universidad de A Coruña, adscrito al Departamento de Análisis Económico y Social Sostenible. Se encuentra realizando en la actualidad su estancia Posdoctoral en la Universidad de A Coruña, en la cual está desarrollando un conjunto de actividades consistentes en dictado de Seminarios, diseño de líneas de investigación y escritura de artículos científicos en referencia al tema de "Financiamiento de las Universidades Públicas para el Desarrollo de sus Misiones para Sociedades Sostenibles". Es autor de los libros "Gestión en la Universidad Pública", "Promoción y Radicación de Inversiones para el Desarrollo de la Producción y el Empleo"; miembro redactor del libro "Mapa de la Discriminación en la Zona Oeste del Gran Buenos Aires"; compilador del libro "Temas de Investigación en Comunicación"; prologuista del libro "Sistema Educativo Argentino. Sus Procesos (Tomo I)" y "La OEA y la Agenda Interamericana"; director del programa de investigación "Comunicación Pública de la Ciencia"; miembro de la Comisión Organizadora de la "Universidad Nacional de La Matanza"; co-Redactor del Estatuto de la Universidad Nacional de La Matanza, en el que se manifiesta la impronta de la Producción Científica con vinculación a la aplicación a la mejora de las condiciones de la Sociedad y creador de los Desarrollos Académicos Científicos y Sociales de la Universidad Nacional de La Matanza.

REDMARKA

UIMA-Universidad de A Coruña - CIECID

Año VIII, Número 14, (2015), v I pp. 103-127

http://www.redmarka.net/

ISSN 1852-2300 
Material original autorizado para su primera publicación en la revista académica REDMARKA. Revista Digital de Marketing Aplicado.

https://doi.org/10.17979/redma.2015.01.014.4883

Recibido: 4 Mayo 2015

Aceptado 15 Junio 2015

\section{RESUMEN}

El presente artículo ha sido realizado sobre la base de la investigación efectuada en el marco de mis estudios de doctorado en Ciencias Económicas en la cual he abordado el estudio de la gestión eficiente de las Universidades Públicas apoyada en las bases teóricas de la productividad, tomando como punto de partida el caso de la Universidad Nacional de la Matanza de la República Argentina. El objetivo es expresar en esta síntesis, los criterios, conceptualizaciones, indicadores e índices que, según los resultados del estudio citado, priorizan la excelencia académica. El método de la investigación aquí abreviada ha sido de carácter cuantitativo correspondiendo a un diseño hipotético deductivo.

La Institución aludida, ha desarrollado desde sus inicios una estructura funcional correspondiente a un modelo alternativo que implica una nueva concepción acerca de la organización y la gestión universitaria. Este nuevo paradigma privilegia una Universidad Pública con Descentralización Académica y Centralización Administrativa.

El desarrollo de medidas que potencien una efectiva y sustentable gestión universitaria y el consenso sobre la forma en que los indicadores la miden, 
constituyen temas de relevancia en agendas de universidades y de gobiernos. La mejora de un sistema de indicadores asociados con resultados de la gestión de las universidades, permite dar respuesta a los imperativos sociales en el marco de políticas públicas.

Presento en este artículo los resultados sintetizados obtenidos en la investigación mencionada, exponiendo la conclusión de los mismos, con la finalidad de abrir líneas de investigación y gestión sobre la base de la eficiencia y la calidad en los estudios universitarios.

PALABRAS CLAVES: UNIVERSIDAD - PRODUCTIVIDAD - EFICIENCIA 


\section{DESARROLLO}

\section{1.- EFICIENCIA Y PRODUCTIVIDAD}

Podemos afirmar que la mayor parte de los sistemas de información especificados por las universidades intentan satisfacer necesidades múltiples, a menudo opuestas y que suponen el vínculo con diversas partes interesadas. Estos intentos de satisfacer el mayor número posible de grupos de interés a menudo, solo producen insatisfacción con los sistemas que finalmente fueron aplicados.

Se abordaron en la investigación, necesidades específicas, aunque bajo reglas internacionalmente aceptadas de modo de facilitar la comparación entre instituciones (Snowball Project, 2011) 2. Las razones conducentes a estos esfuerzos han sido múltiples.

Comparto con Vessuri ${ }^{3}$ que las expectativas en torno de la universidad se ha magnificado y su rol puede resumirse en el denominado "compromiso con el trabajo práctico de la sociedad". Esto significa que además de formar, investigar y transferir para mejorar la posición competitiva de la nación en el mercado mundial y en el desarrollo económico local y regional, esta institución debe dar respuesta a las demandas y necesidades de la sociedad y por lo tanto, el crecimiento de la matrícula deberá contemplar políticas laborales, de movilidad social y de desarrollo territorial. También debe atender a la necesidad de profesionalizar las relaciones institucionales.

Asimismo, al incrementarse la relación de la universidad con el sector productivo, territorial y político, la lógica de sustentabilidad se presenta tan

\footnotetext{
2 The Snowball Project. http://www.projectsnowball.info/wp-content/uploads/snowballproject.pdf ${ }^{3}$ Vessuri, H. (1996) Pertinencia de la educación superior latinoamericana a finales del siglo XX. Nueva Sociedad Nro. 146 Noviembre-Diciembre 1996, pp. 102-107.
} 
relevante como el ingreso, la permanencia y el posicionamiento en los corredores de legitimación disciplinaria y profesional. Las universidades han debido, y deben profesionalizar las relaciones institucionales y generar estructuras y capacidades para asumir nuevas presiones de agentes que desean influir sobre esta sociedad del conocimiento, sean estos gobiernos, industrias o grupos de presión. Otro de los cambios mencionados por Vessuri corresponde a las transformaciones en el plano de la práctica organizacional. Por una parte, la planificación universitaria adquiere rasgos diferentes a la tradicional, mostrándose más flexible y permeable a las condiciones complejas y cambiantes del contexto requiriéndose costos para una multiplicidad de roles lo cual suscita la necesidad de rendir cuentas de la universidad y la de profesionalizar la administración financiera y el fortalecimiento de capacidades en el logro de mayores ingresos, todo ello relacionado con la productividad.

La eficiencia en el manejo de los recursos es vital para las universidades porque hoy, constituye un componente clave de la propia autonomía universitaria. La existencia de estructuras fortalecidas en términos de manejo administrativo de las universidades, resulta imprescindible para amortiguar los efectos de los problemas financieros sobre el devenir de la universidad y sus claustros e, incluso, sobre la declinación de la autonomía intelectual ante la falta de recursos.

Destaco asimismo, la eficiencia como componente de desarrollo estratégico dado el contexto de un entorno económico cambiante y la existencia de nuevas reglas de financiamiento que otorgan importancia especial a la eficacia y la eficiencia la cual se observa como parte de un objetivo estratégico más amplio que el ahorro de costos a corto plazo, donde la calidad y pertinencia constituyen pilares fundamentales de los esquemas innovadores.

Si miramos a los países latinoamericanos, se debe contextualizar el largo proceso de gobiernos militares. En particular, en Argentina ha tomando su máxima expresión en el denominado proceso de reorganización nacional que comienza en

REDMARKA

UIMA-Universidad de A Coruña - CIECID

Año VIII, Número 14, (2015), v I pp. 103-127

http://www.redmarka.net/

ISSN 1852-2300 
1976. En diciembre de 1983 -año en que se recupera el sistema de gobierno democrático, se intervienen las universidades por medio del Decreto 154 por el cual se reimplantan los estatutos con los que se gobernaban las casas de altos estudios antes de 1966. En 1985, se creó el Consejo Interuniversitario Nacional (CIN) con el objetivo de generar un proceso autorregulador del sistema. El retorno de la autonomía generó la construcción de identidades y de un horizonte común al conjunto de instituciones universitarias nacionales. El proceso que se abre en 1983 cumple una primera etapa en 1989, cuando asume el gobierno el Partido Justicialista. El Protocolo para la Concertación universitaria firmado entre el nuevo Presidente de la Nación y los rectores apela a la creciente participación del Ministerio de Educación en la búsqueda de consenso.

Simultáneamente, se establecen las nuevas condiciones materiales y administrativas que han de permitir la estructuración de nuevos mecanismos de coordinación.

El proceso que abre la democratización de la vida del país se caracterizó por la descentralización y desconcentración del sistema de toma de decisiones y de gobierno de las universidades, caracterizadas en la Argentina por su autonomía, en ocasiones mal interpretada. Se verificó fuerte incidencia de la política y el poder económico en la creación de las nuevas universidades.

Con la sanción de la Ley 24.521 Ley Nacional de Educación Superior del 20 de julio de 1995, el análisis de la gestión quedó plasmado en cuatro artículos (15 (Inc. d), 20, 44, y 59).

Las instituciones universitarias se encuentran en la actualidad en proceso de expansión y deben enfrentar constantes cambios. Es por ello que con mucha frecuencia surgen estudios y debates acerca de su funcionamiento.

El desafío es poder resolver asuntos como la burocratización, la politización, la sindicalización del personal, la participación estudiantil, los problemas del financiamiento, la internacionalización académica, la masificación

\author{
REDMARKA \\ UIMA-Universidad de A Coruña - CIECID \\ Año VIII, Número 14, (2015), v I pp. 103-127 \\ http://www.redmarka.net/
}

ISSN 1852-2300 
del ingreso, la búsqueda de nuevas fuentes de recursos y los procesos de informatización de la gestión.

Es claro que a la luz de la complejidad que poseen las instituciones universitarias no es posible gestar un modelo de medición de eficiencia y productividad "inocuo", estrictamente técnico, ajeno a las discusiones políticas y a las estructuras que lo acompañan.

La problemática actual tiene mucho que ver con la inercia burocrática y la adaptación a los nuevos factores que se suceden en un contexto de constantes cambios. Esto termina generando estructuras dispares y que a veces poco tienen que ver con los propósitos básicos de la universidad.

La diversidad académica, el pluralismo institucional y la participación de los estudiantes no deberían ser incompatibles con la existencia de una organización coherente con sus principios y de mecanismos de gestión eficaces.

Lograr una transformación exitosa requerirá un esfuerzo significativo y continuo para vencer resistencias, desmitificar instancias históricamente arraigadas e involucrar a un gran abanico de nuevos actores sociales así como también, contar con el marco tecnológico acorde a la magnitud de tal empresa debido a la necesidad de dar una respuesta orgánica a multitud de problemas científicos y tecnológicos.

Probablemente, por efecto de la permanencia de posiciones encontradas acerca de la eficiencia, por la importante heterogeneidad hacia el interior del sistema universitario o por una combinación de ambas, hasta el presente no se han desarrollado indicadores y mediciones que permitan evaluar la eficiencia y productividad en términos de financiamiento como componente de la calidad.

Si bien comienzan a observarse criterios de evaluación que permitirían el uso intensivo de éstos, el paquete instrumental todavía no los ha incorporado como elemento de evaluación y mejora.

REDMARKA 
Asimismo, existen dos elementos particulares que limitan el uso de mediciones de eficiencia, la heterogeneidad y el tamaño diferente de las distintas instituciones.

La universidad pública argentina ha sido y es evaluada desde distintas perspectivas pero, hay una que no ha sido abordada seriamente, sobre todo a la hora de distribuir los recursos presupuestarios: la eficiencia en la gestión.

La investigación citada, tendió a cubrir ese vacío considerando el análisis de distintas variables para elaborar a nivel teórico y práctico propuestas destinadas a maximizar la productividad y, a partir de una crítica al sistema de distribución de los recursos consignados a educación superior y ofrecer una solución superadora al problema.

Al trabajar el concepto de eficiencia es imprescindible aclarar que no se trata de un ejercicio economicista, y que cada peso gastado en forma ineficiente significa que algún joven está perdiendo la oportunidad de acceder a una formación superior de calidad.

No supone pensar en términos de utilidad económica sino de beneficio social para la Nación toda, que es la que financia con los impuestos que tributan los habitantes de nuestro país el funcionamiento de las universidades estatales, ciudadanos que en su mayoría nunca concurrirán a una universidad pública.

Corregir el sistema de asignación de recursos tiene dos efectos importantes: mejorar la justa asignación de oportunidades y, proporcionar a las instituciones de educación superior un incentivo más adecuado para organizarse institucionalmente y desarrollar sus sistemas de administración y control.

Para lograr eficiencia en la labor académica debemos decidirnos a recorrer un camino que otras instituciones sin fines de lucro han transitado: entender que la eficiencia es un requisito sine qua non para el cumplimiento cabal de la misión que cada una de esas instituciones tiene asignada.

REDMARKA 
Dos conceptos servirán de sostén teórico para el desarrollo de aplicaciones que permitan gestionar las instituciones de Educación Superior: Productividad y Eficiencia.

\section{2.- LOS NUMEROS DE LA EFICIENCIA}

Los esquemas de indicadores aplicados sobre un conjunto de universidades significativas sobre el total de las instituciones universitarias públicas de nuestro país, permitieron analizar la aplicabilidad de los citados conceptos.

Transcribo a continuación los cuadros más significativos del estudio: 
Cuadro № 1: Ejecución presupuestaria total, clasificada por fuente de financiamiento, según Universidad. Su participación porcentual en el total de las fuentes de financiamiento. En pesos.

\begin{tabular}{|c|c|c|c|c|c|c|c|c|c|}
\hline \multirow[t]{3}{*}{ Institución } & \multicolumn{9}{|c|}{ Fuentes de Financiamiento } \\
\hline & \multirow{2}{*}{ Total } & \multicolumn{2}{|c|}{ Tesoro Nacional } & \multicolumn{2}{|c|}{ Recursos Propios } & \multicolumn{2}{|c|}{ Reman.Ej. Anteriores } & \multicolumn{2}{|c|}{ Otras Fuentes } \\
\hline & & en $S$ & $\%$ & en $\$$ & $\%$ & en $\$$ & $\%$ & en $S$ & $\%$ \\
\hline Total de las Instituciones & 9.922 .976 .867 & 8.505 .820 .403 & 85.7 & 756.590 .315 & 7.6 & 488.017 .433 & 4.9 & 172.548 .716 & 1,7 \\
\hline Total Universidades Nacionales & 9.828 .530 .457 & 8.415 .338 .097 & 85,6 & 754.825 .464 & 7,7 & 486.176 .550 & 4,9 & 172.190 .346 & 1,8 \\
\hline Buenos Aires & 2.190 .642 .464 & 1.693 .326 .056 & 77,3 & 326.812 .932 & 14,8 & 149.045 .395 & 6,8 & 21.458 .081 & 1.0 \\
\hline Catamarca & 120.326 .190 & $115.362 .58 \theta$ & 85.8 & 2.507 .710 & 2,1 & 1.082 .361 & 0.8 & 1.373 .550 & 1.1 \\
\hline Centro de la PBA & 167.612 .070 & 146.957 .833 & 87.7 & 13.258 .469 & 7.8 & 6.826 .816 & 4.1 & 588.952 & 0.3 \\
\hline Chaco Austral & 4.763 .072 & $1.138 .69 \theta$ & 23.8 & 3.146 .657 & 68.1 & 477.718 & 10.0 & 0 & 0.0 \\
\hline Chilecito & 28.037 .778 & 22.021 .383 & 78.5 & 40.358 & 0,1 & 2.850 .705 & 10,2 & 3.125 .322 & 11,1 \\
\hline Comahue & 224.118.294 & 213.848 .572 & 85,4 & 2.197 .492 & 1,0 & 6.352 .224 & 2,8 & 1.720 .006 & 0,8 \\
\hline Córdoba & 841.140 .912 & 679.190 .404 & 80,7 & 71.089 .835 & 8,5 & 84.038 .670 & 10.0 & 6.822 .003 & 0.8 \\
\hline Cuyo & 409.802 .937 & 380.952 .479 & 93.0 & 2.015 .390 & 0.5 & 18.709 .311 & 4.6 & 8.125 .757 & 2,0 \\
\hline Entre Rios & 134.818 .019 & 121.389 .988 & 90,0 & 5.857 .512 & 4,4 & 3.898 .335 & 2,8 & 3.572 .184 & 2,6 \\
\hline Formosa & 70.620 .514 & 66.857 .962 & 84.7 & 323.024 & 0.5 & 1.772 .450 & 2.5 & 1.667 .078 & 2.4 \\
\hline Gral. San Martin & 167.237 .977 & 73.399 .681 & 43,8 & 63.683 .033 & 38.1 & 4.614 .159 & 2.8 & 25.561 .104 & 15,3 \\
\hline Gral. Sarmiento & 72.767 .297 & 57.731 .738 & 79.3 & 1.763 .189 & 2,4 & 9.821 .429 & 13,5 & 3.450 .941 & 4,7 \\
\hline Jujuy & 115.286 .178 & 105.285 .689 & 81.3 & 1.344 .826 & 1.2 & 8.675 .653 & 7.5 & 0 & 0,0 \\
\hline La Matanza & 153.145 .660 & 115.830 .913 & 75,6 & 32.276 .384 & 21.1 & 0 & 0.0 & 5.038 .383 & 3,3 \\
\hline La Pampa & 116.475 .316 & 110.188 .679 & 84,8 & 2.831 .758 & 2,4 & 3.446 .246 & 3.0 & 8.633 & 0.0 \\
\hline La Plata & 726.455 .419 & 656.653 .285 & 90,4 & 34.980 .424 & 4,8 & 32.086 .196 & 4,4 & 2.735 .534 & 0,4 \\
\hline La Rioja & 94.801 .302 & 84.687 .607 & 89.3 & 89.730 & 0,1 & 9.459 .345 & 10.0 & 584.620 & 0,6 \\
\hline Lanús & 71.976 .008 & 47.231 .769 & 65.6 & 9.456 .837 & 13,1 & 15.231 .802 & 21.2 & 55.600 & 0.1 \\
\hline Litoral & 300.816 .806 & 272.302 .902 & 90.5 & 23.008 .051 & 7.6 & 4.213 .758 & 1.4 & 1.294 .095 & 0.4 \\
\hline Lomas de Zamora & 167.103 .071 & 136.122 .578 & 81,5 & 17.522 .633 & 10,5 & 13.429 .628 & 8.0 & 28.232 & 0,0 \\
\hline Lujàn & 124.718 .179 & 113.974 .233 & 81.4 & 2.841 .453 & 2,3 & 7.901 .105 & 6.3 & 1.388 & 0.0 \\
\hline Mar del Plata & 233.815 .568 & 218.947 .383 & 93,6 & 5.082 .902 & 2.2 & 9.734 .773 & 4.2 & 70.530 & 0,0 \\
\hline Misiones & 161.412 .001 & 150.243 .045 & 93,5 & 6.617 .888 & 4,1 & 2.709 .172 & 1.7 & 1.141 .896 & 0,7 \\
\hline Nordeste & 328.902 .296 & 280.289 .285 & 85.2 & 30.517 .673 & 9.3 & 0 & 0.0 & 18.095 .358 & 5,5 \\
\hline Noroeste de la PBA & 27.791 .162 & 25.647 .857 & 92,3 & 984.345 & 3.5 & 1.146 .960 & 4,1 & 12.000 & 0,0 \\
\hline Patagonia Austral & 101.787 .896 & 93.530 .402 & 81,8 & 1.836 .733 & 1.8 & 5.248 .567 & 5.2 & 1.172 .184 & 1,2 \\
\hline Patagonia S. J. Bosco & 215.265 .846 & 188.293 .163 & 87.5 & 15.439 .627 & 7,2 & 7.679 .422 & 3.8 & 3.853 .634 & 1,8 \\
\hline Quilmes & 80.200 .333 & 63.280 .151 & 78.8 & 8.991 .902 & 11,2 & 5.283 .044 & 8,8 & 2.645 .236 & 3,3 \\
\hline Rio Cuarto & 175.541 .289 & 167.187 .959 & 95.2 & 3.689 .730 & 2,1 & 4.603 .364 & 2,8 & 60.236 & 0,0 \\
\hline Rio Negro & 23.554 .888 & 23.554 .888 & 100.0 & 0 & 0,0 & 0 & 0.0 & 0 & 0.0 \\
\hline Rosario & 530.074 .934 & 515.406 .616 & 97,2 & 3.536 .640 & 0.7 & 9.907 .754 & 1.8 & 1.223 .924 & 0.2 \\
\hline Salta & 167.845 .630 & 155.937 .530 & $92, \theta$ & 1.323 .176 & 0,8 & 9.687 .908 & 5.8 & 917.016 & 0.5 \\
\hline San Juan & 312.099 .136 & 300.528 .742 & 98.3 & 3.431 .297 & 1.1 & 7.069 .200 & 2,3 & 1.089 .888 & 0.3 \\
\hline San Luis & 176.263 .007 & 187.158 .773 & 94.8 & 1.071 .338 & 0,6 & 7.982 .836 & 4.5 & 50.060 & 0,0 \\
\hline Santiago del Estero & 88.387.977 & 84.786 .971 & 95.8 & 48.000 & 0.1 & 3.236 .820 & 3.7 & 308.186 & 0,3 \\
\hline Sur & 211.855 .513 & 194.335 .336 & 91.7 & 7.371 .303 & 3.5 & 8.408 .920 & 4.0 & 1.739 .854 & 0,8 \\
\hline Tecnológica Nacional (1) & $\ldots$ & $\ldots$ & $\ldots$ & $\ldots$ & $\ldots$ & $\ldots$ & $\ldots$ & $\ldots$ & $\ldots$ \\
\hline Tres de Febrero & 105.902 .528 & 40.382 .717 & 38.1 & 9.775 .214 & 8.2 & 3.311 .575 & 3.1 & 52.453 .022 & 48,5 \\
\hline Tucumán & 540.082 .328 & 491.088 .776 & 90,8 & 36.027 .250 & 8.7 & 12.798 .533 & 2.4 & 187.769 & 0.0 \\
\hline Villa Maria & 45.082 .662 & 39.653 .524 & 88,0 & 1.874 .749 & 4,4 & 3.454 .389 & 7.7 & 0 & 0,0 \\
\hline Total Institutos Universitarios & 94.446 .410 & 90.482 .306 & 95,8 & 1.764 .851 & 1,9 & 1.840 .883 & 1,9 & 358.370 & 0,4 \\
\hline Univ. del Arte & 94.446 .410 & 90.482 .308 & 25.8 & 1.764 .851 & 1,9 & 1.840 .883 & 1.8 & 358.370 & 0,4 \\
\hline
\end{tabular}

\section{REDMARKA}

UIMA-Universidad de A Coruña - CIECID

Año VIII, Número 14, (2015), v I pp. 103-127 http://www.redmarka.net/

ISSN 1852-2300 
Fuente: Anuario de Estadísticas. Secretaría de Políticas Universitarias 2009: Pág 219

REDMARKA

UIMA-Universidad de A Coruña - CIECID

Año VIII, Número 14, (2015), v I pp. 103-127

http://www.redmarka.net/

ISSN 1852-2300 
Cuadro № 2: Indicadores de Población Estudiantil de Instituciones Públicas ${ }^{4}$

\begin{tabular}{|c|c|c|c|c|c|c|c|c|c|c|c|c|c|c|c|c|}
\hline Instuctión & \multicolumn{2}{|c|}{ Estudiantes } & \multicolumn{2}{|c|}{$\begin{array}{c}\text { Nuevos } \\
\text { Imscriptos (ND) }\end{array}$} & \multicolumn{2}{|c|}{ Reinscriptes (RE) } & \multicolumn{2}{|c|}{ Egresados } & $\begin{array}{l}\text { SMMjeres } \\
\text { Estudiantes }\end{array}$ & $\begin{array}{l}\text { SMujeres } \\
\text { Nuevos } \\
\text { Inscriptos }\end{array}$ & $\begin{array}{l}\text { 51Mrieres } \\
\text { Reinseriptos }\end{array}$ & $\begin{array}{l}\text { SMujeres } \\
\text { Epresados }\end{array}$ & $\mid \begin{array}{c}\text { SN } \\
\text { Hasta } 19\end{array}$ & \begin{tabular}{|l|} 
WFE 20 \\
a 24 \\
\end{tabular} & 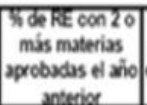 & 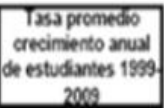 \\
\hline Total de las instituciones "11 & 1,31254 & 1000 & 280.13 & 1000 & 102.412 & 1000 & 69.452 & 1000 & 504 & 55. & 587 & sed & 316 & 307 & 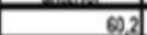 & 22 \\
\hline Tolal Universidades Kacionules & 1.267 .317 & 9.6 & 276.104 & 95.2 & 9月.A13 & 90 & 66.53 & 960 & S6,2 & 55,3 & 36,5 & 59.0 & 322 & 30,7 & का. & 19 \\
\hline Bumes Airss ${ }^{20}$ & 294837 & 225 & 52315 & 18.4 & 241.522 & 23.6 & 10.420 & 23.6 & $\ldots$ & $\ldots$ & $\ldots$ & . & & & es & 0.0 \\
\hline Cxamures & 12127 & 0.8 & 3004 & 1.2 & 8.523 & 0.8 & 316 & 0.5 & 82.8 & 61.8 & 57,7 & 67.4 & 30.5 & 43.6 & 48.0 & 24 \\
\hline Centro de a PQA & 12072 & 1.0 & 2504 & 0.0 & 10.105 & i. & 518 & 0.7 & 80.4 & 52.1 & 80.0 & 57.8 & 48. & 40.5 & 62.8 & 4.1 \\
\hline Choso Austra & 1.000 & 0.1 & 1.040 & 0.7 & . & & . & & $\$, 7$ & 54,7 & . & & 28.0 & & & \\
\hline Crivelio of & 3.67 & 0.3 & 1416 & 0.5 & 2.561 & 0.3 & 20 & 0.0 & 60.8 & 01.2 & 57,8 & 63.8] & 112 & 25.8 & 40.8 & 30.1 \\
\hline Comshus & 20047 & 20 & 7.507 & 20 & 12.140 & 1.0 & 1283 & 1.8 & 68.7 & 68.2 & 60.8 & $e 8.3$ & 28.8 & 11.6 & 40.7 & 1.3 \\
\hline Cinsocos & 103616 & 7.0 & 10.67 & 6.8 & 83010 & 8.2 & 7.241 & 10.4 & 62.2 & 02.6 & 2.1 & es.4 & 523 & 40.7 & s8. & .0 .4 \\
\hline Curo & 31.680 & 24 & 6400 & 2.2 & 28.100 & 25 & 2223 & 3.2 & 028 & 00.2 & ese & 58.2 & 48.1 & 43.7 & 62.7 & 2.8 \\
\hline Erte Rios & 12440 & 0.0 & 2510 & 0.0 & Q.021 & 10 & 1.126 & 1.0 & 61,1 & 62.0 & 60.0 & 74.6 & 50.5 & 43.6 & 58,1 & 1.3 \\
\hline Fomoss & 10.086 & 0.8 & 3326 & 1.1 & 7.630 & 0.7 & 452 & 0.7 & 0,6 & 64.9 & Q6, & 024 & 36.6 & 54.5 & 42,1 & 8.3 \\
\hline Gas. Snn Marin & 11.178 & 0.9 & 3720 & 1,3 & 7.446 & 0.7 & 608 & 1.2 & 00.5 & 88,6 & 61,8 & 61,4 & 13.6 & 33.7 & 67.5 & 12.3 \\
\hline Gra. Srmiento & 4.874 & 0.4 & 766 & 0.3 & 4.086 & 0.4 & 200 & 0.4 & 52.5 & 48,1 & 53.3 & 61.4 & 28.4 & 33.7 & 47.5 & 18.8 \\
\hline Juivy & 12020 & 1.0 & 3008 & 1,1 & 0.871 & 10 & 272 & 0.4 & 86.6 & 57.1 & 80.1 & eid & 27.9 & 32.7 & 30.8 & 2.8 \\
\hline La Notanes & 33348 & 2.5 & 8057 & 28 & 25.291 & 25. & 601 & 12 & 34.0 & 53.5 & 86,3 & 61.2 & 20.7 & 45.7. & 6.8. & 8.0 \\
\hline Laprpa & 8.611 & 0.7 & 2260 & 0.8 & 0.345 & 0.6 & 513 & 0.7 & 60.5 & 63.0 & 80.2 & 60.8 & 40.3 & 47.4 & 65.5 & 20 \\
\hline La Pla & $\infty .167$ & 7.6 & 10.17 & 0.6 & 80.020 & 78 & 4734 & 0.8 & 8.2 & 8.1 & 88.2 & 80.8 & 48. & 28.8 & 52.5 & 1.4 \\
\hline La Rejp & $25.4 \%$ & 1.9 & 4497 & 1.5 & 20.007 & 2.1 & 624 & 0.9 & 68.3 & 53,1 & 60,4 & 68.7 & 43.6. & 430 & 73,1 & 8.0 \\
\hline Lnis & 10.200 & 0.8 & 2328 & 0.8 & 7.881 & 0.8 & 348 & 0.2 & 61,1 & 54,4 & 63.0 & 70.3 & 23.5 & 28.5 & 58.0 & 10.6 \\
\hline Ltora & 40.730 & 3.1 & 8.176 & 3.2 & 31.563 & 3.1 & 1896 & 2.3 & 80.1 & 34.8 & 60.5 & 60.9 & 424 & 43.2 & 63.2 & 5.5 \\
\hline Lomis de 2 mon & 37.212 & 2.8 & 8470 & 20 & 26.742 & 28 & 2320 & 3.4 & 60.8 & 532 & 60.4 & 57.9 & 32.5 & 30.8 & 73.8 & 1.0 \\
\hline Lyjn & 10.980 & 1.3 & 4.110 & 1.4 & 12.470 & 1.2 & 800 & 12 & 6.3 & 60.7 & $\omega, 2$ & eQ.1 & 4.8 & 41,1 & 50.2 & 0.5 \\
\hline Nor del Plas & 21.118 & 1,6 & 3.985 & 1,4 & 17.173 & 1.7 & 1088 & 1.8 & Q4, & 61.6 & 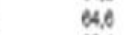 & 61.2 & 230 & 37.8 & 57.6 & .0 .7 \\
\hline Msiones & 20583 & 1,6 & 4.561 & 1.8 & 16.032 & 1.0 & 607 & 1.0 & 00.4 & 00.1 & 60.4 & e3.1] & 43.3 & 45.5 & 40.2 & 2.9 \\
\hline Norbeste & 50.800 & 3.0 & 12323 & 42 & 38.567 & 3.8 & 3002 & 4.4 & 88,1 & 53.1 & 88,8 & 01,3 & 42.1 & 30.5 & 73.7 & 0.1 \\
\hline 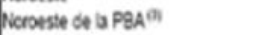 & 8.136 & 0.4 & 1.808 & 0.6 & 3330 & 0.3 & 31 & 0.0 & 80.6 & 52.0 & 40.5 & 64.5 & 30.5 & 63.6 & 50.0 & 3.1 \\
\hline Pxaponia Austral & 0.362 & 0.5 & 2712 & 0.0 & 3060 & 0.4 & 173 & 0.2 & eQ.5 & 68.0 & 70,5 & 20.8 & 13.5 & 30.0 & 43.2 & 3.8 \\
\hline Praponia S. J. Bosso & 13686 & 1.0 & 3.865 & 1,3 & $0.7 \pi 1$ & 10 & 517 & 0.7 & 026 & 61,4 & es.1 & 61,7 & 37. & 40.3 & 43.7 & 1,1 \\
\hline Quimes & 12762 & 1.0 & 3810 & 1,3 & 8.043 & 0.0 & 718 & 1.0 & 80.0 & 00.1 & 68,5 & e6.3. & 12.4 & 20.3 & 80.8 & 10.8 \\
\hline Rio Cuaro & 18.138 & 1.2 & 3.101 & 1,1 & 13.037 & 1.3 & 880 & 1.2 & 57.4 & 58.6 & 57.2 & 62.5 & 300 & 524 & 00.0 & 1,3 \\
\hline Ro Negro & 1022 & 0.1 & 1022 & 0.0 & & & . & & 30.8 & 80.8 & . & & 11,1 & & & \\
\hline Rossio & 71.547 & 3.5 & 13060 & 4.7 & 68.157 & 8.7) & 6.877 & Q.8 & 00.8 & 00.0 & 61,0 & 34.6 & 53.5 & 45. & 54.1 & 0.7 \\
\hline sata & 23260 & 1.8 & 4.967 & 1.6 & 18.702 & 1.8 & 133 & 0.2 & 82.0 & 58.4 & 88,6 & 34.9 & 38.4 & 38.3 & 30.2 & 3.0 \\
\hline $\sin \operatorname{Jan}$ & 17.822 & 1.4 & 3.688 & 1,3 & 13.657 & 1.4 & 887 & 0.2 & 80.2 & 88.4 & 30.5 & 50.4 & 40.4 & 48.5 & 58.2 & 20 \\
\hline Sintus & 11.974 & 0.8 & 3006 & 1,1 & 8.500 & 0.0 & 020 & 0.9 & 60.3 & 60.5 & es, 3 & $e 8,3$ & 30.7 & 42. & G.4 & $-1,4$ \\
\hline Santapo del Estero & 14723 & 1.1 & 4.172 & 1,4 & 10.861 & 1.0 & 802 & 1.2 & eQ.1 & 74.1 & 67,1 & 83. & 230 & 20.2 & 51,1 & 3.0 \\
\hline sur the the & 18.840 & 1.4 & 478 & 1.8 & 14.108 & 1,4 & 1000 & 1.4 & 88.4 & s.1 1 & 68,5 & 57.5 & 6.7. & 56.0 & 67,4 & 0.7 \\
\hline Teenolicaca Nacional & 75.000 & 3.8 & 18062 & 0.2 & 57.808 & 5.7 & 4.171 & o.d & 20.4 & 21.8 & 20.0 & 28.0 & 36.0 & 40.5 & 65.0 & 1.8 \\
\hline Ties de febreo & 8.714 & 0.7 & 4.352 & 1.5 & 4.362 & 0.4 & 268 & 0.4 & 47,3 & 430 & 31,5 & e2.7 & 120 & 28.6 & 712 & 10.2 \\
\hline Tuoumin & 00,48 & 4.0 & 12224 & 42 & 46.424 & 4.7) & 1.860 & 28] & 01,1 & 61.1 & 61,1 & $e 0.0$ & 40.0 & 4.2 & 50.1 & 20 \\
\hline Vila Moria & 4000 & 0.3 & 154 & 0.5 & 2.555 & 0.2 & 130 & 0.2 & 578 & 020 & 88,3 & 57.0 & 25.1 & 41.7 & 57,5 & 11,0 \\
\hline Total Insthtuos Universitarios & 22.155 & 2.2 & 8.872 & 3,1 & 20.283 & $20 \mid$ & 2002 & 29 & 57,4 & 56,0 & 58,0 & 49.3 & 15.4 & 25.1 & 73.6 & 20.3 \\
\hline & Q.0.45 & 0.5 & 1.088 & 0.6 & 5.200 & 0.5 & 762 & 1,1 & 45.6 & 43.7 & 48.2 & 528 & 30.3 & 37,7 & 68.6 & 8.8 \\
\hline Enseinsa Supeior del Etroto & 1.452 & 0.1 & 48 & 0.2 & eat & 0.1 & $2 n$ & 0.4 & 8.8 & 12.5 & 7,1 & 5.8 & 500 & 020 & M.E. & 3.5 \\
\hline & 1.00 & 0.1 & 327 & 0.1 & $m$ & 0.1 & 178 & 0.3 & 10,6 & 220 & 14,2 & 12.4 & 48.6 & 3.5. & 42.1 & 7.4 \\
\hline Paicia Feseral Anpertins & 4000 & 0.4 & 2501 & 0.0 & 2.465 & 0.2 & 362 & 0.5 & 86.7 & 54.7 & 60.7 & 60.5 & 8.0 & 35.4 & 80.4 & 0.8 \\
\hline Seguidas Maritim & $2 * 0$ & 0.0 & 131 & 0.0 & 132 & 0.0 & . & & 16,7 & 10.1 & 14,4 & & 420 & 87. & 88.6 & \\
\hline insthato Univ de Ane & 14.400 & 1,1 & $37 \pi 0$ & 1,3 & 10.250 & 10 & 433 & 0.6 & 72.5 & 72.1 & 72,7 & 70,7 & 5.5. & 10.1 & 73.6 & 0.2 \\
\hline Total Universidad Provincial & 15.87 & 1,2 & 5.161 & 1,8 & 10.716 & 1.0 & 797 & 1.1 & 65,8 & 64,1 & 66,7 & 74,4 & 25.1 & 42.11 & ses. & 19.1 \\
\hline Autoinonu de Entre Rios & 15.877 & 12 & 5101 & 18 & 10.716 & 10 & 797 & 1,1 & 68 & 64.1 & 607 & 744 & 2501 & 42,1 & s.s & 10.1 \\
\hline
\end{tabular}

${ }^{4}$ http://repositorio.educacion.gov.ar:8080/dspace/bitstream/handle/123456789/66203/Anuario-

2009.pdf?sequence $=1$

REDMARKA

UIMA-Universidad de A Coruña - CIECID

Año VIII, Número 14, (2015), v I pp. 103-127

http://www.redmarka.net/

ISSN 1852-2300 
Cuadro № 3: Cantidad de Alumnos (Años 2007-2008-2009)

\begin{tabular}{|l|c|c|c|}
\hline \multirow{2}{*}{\multicolumn{1}{|c|}{ UNLaM y TOTAL }} & \multicolumn{3}{c|}{ Cantidad de Alumnos } \\
\cline { 2 - 4 } & 2007 & 2008 & 2009 \\
\hline Universidad Nacional de La Matanza & 30.276 & 32.378 & 33.348 \\
\hline Totales UUNN & 1.242 .838 & 1.255 .714 & 1.267 .517 \\
\hline
\end{tabular}

El crecimiento del total de alumnos de las Universidades Nacionales en los años 2007, 2008 y 2009 fue del 1.98\%, mientras que la UNLaM ha crecido en cantidad de alumnos el $10.15 \%$.

Cuadro № 4: Cantidad de Docentes (Años 2007-2008-2008)

\begin{tabular}{|l|c|c|c|}
\hline \multirow{2}{*}{ UNLaM y TOTAL } & \multicolumn{3}{c|}{ Cantidad de Docentes $^{*}$} \\
\cline { 2 - 4 } & 2007 & 2008 & 2009 \\
\hline Universidad Nacional de La Matanza & 1.485 & 1.579 & 1.671 \\
\hline Totales UUNN & 164.411 & 171.421 & 107.350 \\
\hline "Hasta 2008 se informan nummeros de cargos docentes & \multicolumn{3}{|l}{} \\
\hline
\end{tabular}

Cuadro № 5: Cantidad de Egresados (Años 2007-2008-2008)

\begin{tabular}{|l|c|c|c|}
\hline \multirow{2}{*}{\multicolumn{1}{|c|}{ UNLaM y TOTAL }} & \multicolumn{3}{c|}{ Cantidad de Egresados } \\
\cline { 2 - 4 } & 2007 & 2008 & 2009 \\
\hline Universidad Nacional de La Matanza & 144 & 110 & 268 \\
\hline Totales UUNN & 60.348 & 62.693 & 66.653 \\
\hline
\end{tabular}

El crecimiento del total de Universidades Nacionales de egresados fue del $10.45 \%$, mientras que la UNLaM ha crecido en cantidad de alumnos el $10.09 \%$.

REDMARKA

UIMA-Universidad de A Coruña - CIECID

Año VIII, Número 14, (2015), v I pp. 103-127

http://www.redmarka.net/

ISSN 1852-2300 
Cabe consignar que los datos referidos a egresados no ofrecen una comparación válida, ya que buena parte de las instituciones universitarias, comenzando por la UBA, contabilizan como egresados a aquellos alumnos que obtienen un título intermedio. Este hecho hace que las comparaciones no tengan validez alguna. La UNLaM solo ha contabilizado los alumnos que han obtenido un título de grado.

Cuadro № 6: Cantidad de Ingresantes (Años 2007-2008-2008)

\begin{tabular}{|l|c|c|c|}
\hline \multirow{2}{*}{\multicolumn{1}{|c|}{ UNLaM y TOTAL }} & \multicolumn{3}{c|}{ INGRESANTES } \\
\cline { 2 - 4 } & 2007 & 2008 & 2009 \\
\hline Universidad Nacional de La Matanza & 4.772 & 4.354 & 8.057 \\
\hline Totales UUNN & 262.638 & 261.809 & 276.104 \\
\hline
\end{tabular}

Con respecto a la cantidad de ingresantes, el crecimiento del total de Universidades Nacionales fue del $5.13 \%$, mientras que la UNLaM ha crecido en cantidad de Alumnos Ingresantes el 68.84\%.

Cuadro № 7: Cantidad No Docentes (Años 2007-2008-2008)

\begin{tabular}{|l|r|r|r|}
\hline \multirow{2}{*}{ UNLaM y TOTAL } & \multicolumn{3}{c|}{ No docentes } \\
\cline { 2 - 4 } \multicolumn{1}{|c|}{} & 2007 & 2008 & 2009 \\
\hline Universidad Nacional de La Matanza & 316 & 352 & 368 \\
\hline Totales UUNN & 44.301 & 45.065 & 43.830 \\
\hline
\end{tabular}

Las Universidades Nacionales han decrecido el $1.06 \%$ en cantidad de No Docentes, mientras que la UNLaM creció el $16.45 \%$.

REDMARKA

UIMA-Universidad de A Coruña - CIECID

Año VIII, Número 14, (2015), v I pp. 103-127

http://www.redmarka.net/

ISSN 1852-2300 
Cuadro № 8: Presupuesto Asignado en Millones de Pesos

(Años 2007-2008-2008)

\begin{tabular}{|l|c|c|c|}
\hline \multirow{2}{*}{\multicolumn{1}{|c|}{ UNLaM y TOTAL }} & \multicolumn{3}{|c|}{ PRESUP. ASIGNADO (en millones } \\
\cline { 2 - 4 } & 2007 & 2008 & 2009 \\
\hline Universidad Nacional de La Matanza & 96 & 117 & 167 \\
\hline Totales UUNN & 6.191 & 8.823 & 11.793 \\
\hline
\end{tabular}

En cuanto al presupuesto asignado, es de notar que el total de Universidades Nacionales creció el $90.48 \%$, mientras que el de la UNLaM creció el $73.96 \%$.

Cuadro № 9: Cantidad de Alumnos por Docente (Años 2007-2008-2008)

\begin{tabular}{|l|c|c|c|}
\hline \multirow{2}{*}{\multicolumn{1}{|c|}{ UNLaM y TOTAL }} & \multicolumn{3}{c|}{ ALUMNOS POR } \\
\cline { 2 - 4 } & 2007 & 2008 & 2009 \\
\hline Universidad Nacional de La Matanza & 20 & 21 & 20 \\
\hline Totales UUNN & 10 & 9 & 13 \\
\hline
\end{tabular}

* Hasta 2008 se informan № de cargos docentes

La Cantidad de Alumnos por Docente en la UNLaM fue del $0 \%$, mientras que en las Universidades Nacionales creció el 30\%.

Cuadro № 10: Cantidad de Alumnos por No Docente (Años 2007-2008-2008)

REDMARKA

UIMA-Universidad de A Coruña - CIECID

Año VIII, Número 14, (2015), v I pp. 103-127

http://www.redmarka.net/

ISSN 1852-2300 


\begin{tabular}{|l|c|c|c|}
\hline \multirow{2}{*}{\multicolumn{1}{|c|}{ UNLaM y TOTAL }} & \multicolumn{3}{|c|}{$\begin{array}{c}\text { ALUMNOS POR NO } \\
\text { DOCENTE }\end{array}$} \\
\cline { 2 - 4 } & 2007 & 2008 & 2009 \\
\hline Universidad Nacional de La Matanza & 96 & 92 & 91 \\
\hline Totales UUNN & 37 & 35 & 36 \\
\hline
\end{tabular}

En relación a la cantidad de Alumnos por No Docente, las Universidades Nacionales cayeron el $2.70 \%$ y la UNLaM cayó el $5.21 \%$.

Cuadro № 11: Presupuesto por Alumno (Años 2007-2008-2008)

\begin{tabular}{|l|c|c|c|}
\hline \multirow{2}{*}{\multicolumn{1}{|c|}{ UNLaM y TOTAL }} & \multicolumn{3}{|c|}{ PRESUPUESTO POR } \\
\cline { 2 - 4 } & 2007 & 2008 & 2009 \\
\hline Universidad Nacional de La Matanza & 3171 & 3614 & 5008 \\
\hline Totales UUNN & 5.995 & 7.597 & 10.474 \\
\hline
\end{tabular}

En promedio el presupuesto por Alumno en las Universidades Nacionales creció el $74.71 \%$, mientras que en la UNLaM creció el $57.93 \%$.

Cuadro № 12: Presupuesto por Egresado en Pesos (Años 2007-2008-2008)

\begin{tabular}{|c|c|c|c|}
\hline \multirow{2}{*}{ UNLaM y TOTAL } & \multicolumn{3}{|c|}{ PRESUPUESTO POR } \\
\cline { 2 - 4 } & 2007 & 2008 & 2009 \\
\hline Universidad Nacional de La Matanza & 123.552 & 143.911 & 208.489 \\
\hline Totales UUNN & 164.219 & 238.560 & 300.598 \\
\hline
\end{tabular}

\section{REDMARKA}

UIMA-Universidad de A Coruña - CIECID

Año VIII, Número 14, (2015), v I pp. 103-127

http://www.redmarka.net/

ISSN 1852-2300 
En cuanto al presupuesto por Alumno, el promedio de las Universidades Nacionales creció el $83.05 \%$ y la UNLaM el 68.74\%.

\section{3.- EL CASO UNLAM}

El caso referenciado en el título se plantea como caso capaz de verificar los constructos teóricos propuestos para el desarrollo de la gestión eficiente.

A los efectos de establecer una comparación, los datos presentados en los cuadros precedentes muestran la evolución de algunos valores en términos absolutos de ciertos indicadores de eficiencia de la UNLaM y del total de un grupo de universidades significativas de la totalidad del sistema universitario argentino. El análisis se muestra en términos porcentuales.

El indicador más claro es el de presupuesto por alumno, que sitúa a la UNLaM en el primer puesto en cuanto a grado de eficiencia, ya que para 2009 recibió $\$ 5.008$ por alumno, la mitad del promedio del sistema que fue de $\$ 10.474$, y absolutamente distanciada de Universidades como la de San Juan, que obtuvo \$ 18.573 por alumno, Cuyo, \$16.141, San Martín, \$15.839, o Tres de Febrero, \$ 14.804, todas para el mismo período. El significado real de esta comparación es que si cada una de las universidades citadas tuviera un grado de eficiencia equivalente al de la UNLaM, deberían estar formando a un número de alumnos tres veces mayor que el que atienden en la actualidad.

$\mathrm{Si}$ analizamos la cantidad de alumnos por personal administrativo, nuevamente aparece la UNLaM a la cabeza con 91 alumnos por administrativo, contra 36 del promedio del sistema.

No es posible considerar con precisión el número de alumnos por docentes ya que la fuente consultada no presenta datos de docentes distribuidos por designación y las dedicaciones docentes pueden abarcar seis niveles que van desde una dedicación simple hasta una dedicación exclusiva. En cuanto a la 
Universidad Nacional de La Matanza, es necesario tener en cuenta que esta Universidad duplica la proporción de dedicaciones exclusivas del sistema.

También, para comprender la magnitud de la situación planteada, si todo el sistema funcionara con el nivel de eficiencia de la UNLaM, se podría formar al doble de alumnos universitarios a nivel nacional, lo cual significa 1.300 .000 alumnos más (cifras para el año 2009).

Crítica al sistema de distribución de los recursos destinados a educación superior para ofrecer una solución superadora al problema.

El sistema propuesto por el CIN y aceptado por la Secretaría de Políticas Universitarias, parte de un algoritmo que recrea lo que sucede en el conjunto del sistema universitario público. Dicho sistema ha sido implementado solo parcialmente (otros gastos), y sin periodicidad ni uniformidad.

\section{1.- CRITERIOS ESTABLECIDOS POR EL MODELO CIN}

De acuerdo con los criterios establecidos por el modelo propuesto por el Consejo Interuniversitario Nacional y, con el fin de alcanzar indicadores que sean representativos para un determinado período, se han tomado en cuenta los datos registrados en el Anuario de Estadísticas Universitarias de la Secretaría de Políticas Universitarias del Ministerio de Educación de la Nación publicado en el año 2011, con datos oficiales correspondientes a 2009.

El Modelo aprobado por el AP 465/03 se sustenta en los siguientes criterios: 1) la "función objetivo" como voluntad estratégica de alcanzar su cumplimiento, consistente en que las Universidades Nacionales no tengan un compromiso mayor al $75 \%$ de su presupuesto total asignado personal docente y no docente y el $25 \%$ restante a otros gastos; 2) el concepto de "alumno referenciado", el que partiendo de los alumnos nominales de cada universidad, son ajustados independientemente por un índice de Economía de Escala, un índice de Actividad 
Académica y un índice de Complejidad de la Oferta Académica; 3) el Modelo Normativo de la SPU; 4) la relación entre la categoría de los investigadores y la dedicación de cada docente categorizado y 5) la extensión la cual no se considera conveniente su incorporación, atento a que no pueden establecerse indicadores comunes, ya que las funciones de distintas universidades difieren según su ámbito geopolítico.

En cuanto a la metodología para distribuir los fondos adicionales, se resuelve para el año 2003 las siguientes proporciones: 1ำ distribución: $45 \%$ en función del tamaño de cada universidad (Economía de Escala Global), corresponde al b \% del AP 395/01; 2ำ distribución: $5 \%$ para programas de investigación. Corresponde al d\% del AP 395/01; 3ํdistribución: 50 \% en función de la metodología del Presupuesto Normativo de la SPU. Corresponde al c \% del AP 395/01.

La suma de estas tres distribuciones será el incremento presupuestario que le corresponderá a cada universidad del total. $(b \%+c \%+d \%)$

\title{
3.1. 1.- Alumno Referenciado
}

La variable alumno es una de las fundamentales de cualquier sistema de costos (y presupuestos) educativos. El Modelo de Pautas Objetivas de Distribución Presupuestaria del CIN así lo reconoce expresamente, y para ello, construye la propuesta sobre la base de la homogeneización de los alumnos nominales (observados) de cada universidad nacional a través de 3 sucesivos ajustes: 1 ) Ajuste por Índice de Economía de Escala Global consistente en tener en cuenta la existencia de economías de escala globales; 2) Ajuste por Índice de Complejidad de la Oferta Académica correspondiente al número de estudiantes universitarios cursando carreras, (suma de los estudiantes inscriptos en primer año corregidos por un factor de deserción más los inscriptos que hayan aprobado dos o más

\author{
REDMARKA \\ UIMA-Universidad de A Coruña - CIECID \\ Año VIII, Número 14, (2015), v I pp. 103-127 \\ http://www.redmarka.net/
}

ISSN 1852-2300 
asignaturas en el ciclo lectivo del año anterior hasta el 31/12/año anterior); y 3) Ajuste por el Índice de Actividad Académica el cual recoge la influencia de la demanda presupuestaria diferencial que tienen las distintas carreras que se dictan en el Sistema Universitario Nacional.

El valor para los alumnos referenciados de la UNLaM se obtuvo por la suma de los alumnos declarados nominales (22.990), los alumnos referenciados por IEE (16.392), los alumnos referenciados por ICOA (7580) y los alumnos referenciados por IAA (0). El total obtenido es 46.962.

3.1.2.- Determinación de la planta de personal no docente.

El Modelo adopta una metodología para el caso de una Universidad con estructura de facultades, desagregando cada dotación de acuerdo al esquema que se indica más adelante. La UNLaM, por su estructura departamental, tiene una administración centralizada. Esto hace que varias de las funciones que son propias de cada facultad o unidad académica, estén unificadas dentro de la administración de la institución. Así, los Departamentos (Unidades Académicas) tienen dotaciones mínimas de personal no docente, para realizar sus tareas que mayormente delega a la administración de la Universidad. De este modo, resulta una mejor articulación tanto horizontal como vertical.

La administración centralizada disminuye costos en tanto evita duplicaciones esencialmente de costos fijos. Tener una estructura de administración de personal en cada facultad (esquema descentralizado) produce duplicaciones de funciones que perfectamente pueden evitarse al centralizar la función.

\subsubsection{Dotaciones para Rectorado y Actividades Centrales}

REDMARKA 
Según la normativa del modelo, se plantea una estructura mínima de 60 cargos no docentes, para una Universidad de 3000 alumnos y 3 facultades. A partir de esa estructura mínima se plantea un ajuste por cantidad de alumnos y un ajuste por cantidad de unidades de gestión a vincular y coordinar.

La UNLaM tenía al momento del análisis 313 personas trabajando en sus distintas áreas. El total de dotación para rectorado y actividades centrales según normativa es de 212 .

En UNLaM, teniendo en cuenta la dotación por departamentos según normativa adquiere los siguientes valores: Departamento de Ciencias Económicas, 87, Departamento de Ingeniería e Investigaciones Tecnológicas, 67; Departamento de Humanidades y Ciencias Sociales, 67 y Departamento de Derecho y Ciencia Política: 47.

\subsection{4.- Personal no docente:}

En UNLaM, teniendo nuevamente en cuenta la dotación por departamentos según normativa adquiere los siguientes valores: Departamento de Ciencias Económicas, 10, Departamento de Ingeniería e Investigaciones Tecnológicas, 10; Departamento de Humanidades y Ciencias Sociales, 10 y Departamento de Derecho y Ciencia Política: 5.

\subsection{5.- Personal de Servicios y Mantenimiento:}

Se toma este valor según variación por metros cuadrados edificados. La normativa indica 1 agente por cada 500 metros cuadrados edificados, resultando para UNLaM 116 agentes. Según ajuste de no docentes por metros cuadrados

\section{REDMARKA}


edificados y teniendo en cuenta la referencia media metros cuadrados por alumno: 6. El caso UNLaM es de 1,24. Es decir que no ajusta. En cuanto a la variación por metros cuadrados de espacio verde, según la normativa se calcula 1 Agente por cada 30.000 metros cuadrados de espacio verde. El valor correspondiente a UNLaM es de 8.

Teniendo en cuenta las variables totales, según la normativa el valor es de 124 y el de la UNLaM es de 49.

De todo lo expuesto, surge que si aplicáramos el Modelo del CIN a la estructura organizativa de la Universidad Nacional de La Matanza, deberíamos incrementar la planta de personal no docente en un $56 \%$, lo cual da una idea concreta del grado de eficiencia que la citada institución ha alcanzado.

\section{CONCLUSIONES}

El nivel de eficiencia que nos lleva a analizar, tanto en lo teórico como en lo práctico, la estructura de la UNLaM permite trasladar la experiencia de una eficiente aplicación de los recursos que la sociedad le provee, a la maximización de los beneficios sociales que la Universidad genera a partir del logro de un alto nivel de productividad en la aplicación de los citados recursos.

Paralelamente al cuadro de asimetría, la alta dependencia de las universidades estatales al presupuesto público podría estar restringiendo la utilización de mediciones asociadas con su manejo y eficiencia en tanto que, por la autonomía consagrada en sucesivas leyes universitarias, los modelos de gestión de las universidades son establecidos bajo reglas propias.

El dar cuenta de resultados de eficiencias, en rigor, lo que pone a la luz es la opción de gestión y estructura por la que han optado cada una de las instituciones que conforman el sistema universitario.

REDMARKA

UIMA-Universidad de A Coruña - CIECID

Año VIII, Número 14, (2015), v I pp. 103-127

http://www.redmarka.net/

ISSN 1852-2300 
Esta doble limitación conduce a la necesidad de gestar modelos de medición de la eficiencia y productividad ampliados, esto es, comprensibles en términos de indicadores de eficiencia universitaria así como en términos de características estructurales y organizacionales, en tanto que las instituciones universitarias se encuentran con grandes desafíos en la actualidad para poder resolver asuntos como la burocratización, la politización, la sindicalización del personal, la participación estudiantil, los problemas del financiamiento, la internacionalización académica, la masificación del ingreso, la búsqueda de nuevas fuentes de recursos y los procesos de informatización de la gestión.

Es claro que a la luz de la complejidad que poseen las instituciones universitarias no es posible gestar un modelo de medición de eficiencia y productividad "inocuo", estrictamente técnico, ajeno a las discusiones políticas y a las estructuras que lo acompañan.

La problemática actual tiene mucho que ver con la inercia burocrática y la adaptación a los nuevos factores que se suceden en un contexto de constantes cambios. Esto termina generando estructuras dispares y que a veces poco tienen que ver con los propósitos básicos de la universidad. La diversidad académica, el pluralismo institucional y la participación de los estudiantes no deberían ser incompatibles con la existencia de una organización coherente con sus principios y de mecanismos de gestión eficaces. Lograr una transformación exitosa requerirá un esfuerzo significativo y continuo para vencer resistencias, desmitificar instancias históricamente arraigadas e involucrar a un gran abanico de nuevos actores sociales.

Se propone optimizar la distribución presupuestaria a partir de considerar los niveles de eficiencia alcanzados por cada universidad para asignar partidas crecientes a aquellas instituciones que hayan logrado incrementos de la productividad en su gestión. 


\section{BIBLIOGRAFÍA}

Abbott, M. and Doucouliagos, C. (2003) "The Efficiency of Australian Universities: A Data Envelopnient Analysis", Economías of Education Review, 22.

Asís, D. (2007) Análisis de eficiencia de los departamentos universitarios. El caso de la Universidad de Sevilla. Librería-Editorial Dykinson.

Clark, Burton R. (1992) El sistema de educación superior: una visión comparativa de la organización académica. México. UAM.

CONEAU. (1997). Lineamientos para la evaluación institucional. Buenos Aires: Ministerio de Cultura y Educación.

Coria, M. (2008) Eficiencia técnica de las universidades de gestión estatal en Argentina. Pontificia Universidad Católica Argentina "Santa María de los Buenos Aires" - Facultad de Ciencias Sociales y Económicas, Departamento de Economía. Documento de Trabajo $\mathrm{N}^{\circ} 27$.

Etkin, Jorge Ricardo; Schvarstein, Leonardo. (1993). Identidad de las organiLzaciones: invariancia y cambio. Buenos Aires. Paidós.

Laurent, J.; Fregosi, A.; Laguzzi, H. (1973). Organización departamental: documentos para una reforma universitaria. Buenos Aires. CRUN.

Meneses Morales, Ernesto (1971). "La organización departamental en las universidades". México: Revista del Centro de Estudios Educativos. VI, №3, 1971.

McMaster, M (2010) Partnerships between Administrative and Academic

Mintzberg, Henry; Quinn James Brian; Voyer, John (1997) El proceso estratégico: conceptos, contextos y casos. México. Prentice-Hall.

(1991) Diseño de organizaciones eficientes. Buenos Aires, El Ateneo.

Secretaría de políticas universitarias (1998) La educación Superior en Argentina: una visión compartida. Buenos Aires. Ministerio de Cultura y Educación.

The Snowball Project. Acceso y disponibilidad:

<http://www.projectsnowball.info/wp-content/uploads/snowballproject.pdf>

REDMARKA

UIMA-Universidad de A Coruña - CIECID

Año VIII, Número 14, (2015), v I pp. 103-127

http://www.redmarka.net/

ISSN 1852-2300 
Vessuri, H. (1996) Pertinencia de la educación superior latinoamericana a finales del siglo XX. Nueva Sociedad Nro. 146 Noviembre-Diciembre 1996, pp. 102-107. 